University of Wollongong

Research Online

Faculty of Engineering - Papers (Archive)

Faculty of Engineering and Information

Sciences

$1-1-2005$

\title{
Multi-objective evolutionary algorithm for investigating the trade-off between pleiotropy and redundancy
}

\author{
Zhiyang Ong \\ University of Adelaide \\ Hao-Wei Lo \\ University of Adelaide \\ Matthew J. Berryman \\ University of Wollongong, mberryma@uow.edu.au \\ Derek Abbott \\ University of Adelaide, dabbott@eleceng.adelaide.edu.au
}

Follow this and additional works at: https://ro.uow.edu.au/engpapers

Part of the Engineering Commons

https://ro.uow.edu.au/engpapers/4902

\section{Recommended Citation}

Ong, Zhiyang; Lo, Hao-Wei; Berryman, Matthew J.; and Abbott, Derek: Multi-objective evolutionary algorithm for investigating the trade-off between pleiotropy and redundancy 2005.

https://ro.uow.edu.au/engpapers/4902

Research Online is the open access institutional repository for the University of Wollongong. For further information contact the UOW Library: research-pubs@uow.edu.au 


\title{
Multi-objective evolutionary algorithm for investigating the trade-off between pleiotropy and redundancy
}

\author{
Zhiyang Ong ${ }^{a, c}$, Andy Hao-Wei Lo ${ }^{a}$, Matthew Berryman ${ }^{a, b}$, and Derek Abbott ${ }^{a, b}$ \\ ${ }^{a}$ School of Electrical and Electronic Engineering, and \\ ${ }^{b}$ Centre for Biomedical Engineering, \\ University of Adelaide, Adelaide SA 5005, Australia; \\ ${ }^{c}$ Department of Electrical Engineering, University of Southern California, CA 90089-2560, USA
}

\begin{abstract}
The trade-off between pleiotropy and redundancy in telecommunications networks is analyzed in this paper. They are optimized to reduce installation costs and propagation delays. Pleiotropy of a server in a telecommunications network is defined as the number of clients and servers that it can service whilst redundancy is described as the number of servers servicing a client. Telecommunications networks containing many servers with large pleiotropy are cost-effective but vulnerable to network failures and attacks. Conversely, those networks containing many servers with high redundancy are reliable but costly. Several key issues regarding the choice of cost functions and techniques in evolutionary computation (such as the modeling of Darwinian evolution, and mutualism and commensalism) will be discussed, and a future research agenda is outlined. Experimental results indicate that the pleiotropy of servers in the optimum network does improve, whilst the redundancy of clients do not vary significantly, as expected, with evolving networks. This is due to the controlled evolution of networks that is modeled by the steady-state genetic algorithm; changes in telecommunications networks that occur drastically over a very short period of time are rare.
\end{abstract}

Keywords: Complex systems, evolutionary algorithm, network optimization, pleiotropy, redundancy

\section{INTRODUCTION}

Complex systems can be modeled as a network, whereby the interactions between the systems' components define their overall behavior and functions. By analyzing each entity, their emergent behaviour and self-organized structures cannot be determined. Each complex system may also have nested complex networks, which may have its own embedded com-

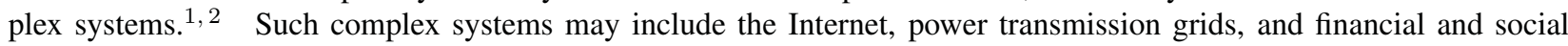
networks. ${ }^{3,4}$ Thus, by modeling and examining the properties of a simple telecommunications network without network routing and data packet transmission as a complex system, where each node may represent a smaller communications network, telecommunications companies may be able to improve the reliability and reduce the cost of the networks.

The pleiotropy of a server in a telecommunications network is defined as the number of clients that it can attend to, whilst the redundancy is described as the number of servers servicing a client. ${ }^{5,6}$ A client-server network with a mixture of pleiotropy and redundancy can be seen in Fig. 1. This indicates good robustness in the system; if server A and C fail, the set of clients $\{1,2,3,4\}$ can still function with the service of server B. On the other hand, allowing the server (for example, server B in Fig. 1) to serve more than one client reduces the costs of installing expensive servers. Consequently, network topologies can be optimized so that the networks can be both robust and have low setup or runtime costs.

An abundance of methods exist for network optimization, however, steady state evolutionary algorithms are used since they can model the incremental growth of the telecommunications networks as an organic process. ${ }^{5,6}$ They allow us to capture the dynamic of the telecommunications network as data links, servers, and clients are added to the network, removed from it, fail, or get repaired. Thus, the modification of the problem specifications, constraints, and/or objective functions do not require the optimization process to be restarted as evolutionary algorithms can adapt to the changes. Moreover, evolutionary algorithms search for a solution from a diverse set of solution space, as opposed to searching from a starting point. ${ }^{7,8}$ Since a guaranteed optimal solution is not provided by evolutionary algorithms, they are used as a tool in the design process as opposed to a tool for determining the optimum topology of a telecommunications network. ${ }^{9}$

Further author information: (Send correspondence to Zhiyang Ong and Derek Abbott) E-mail: zhiyang@ieee.org, dabbott@eleceng.adelaide.edu.au; Telephone: +61 883035748 


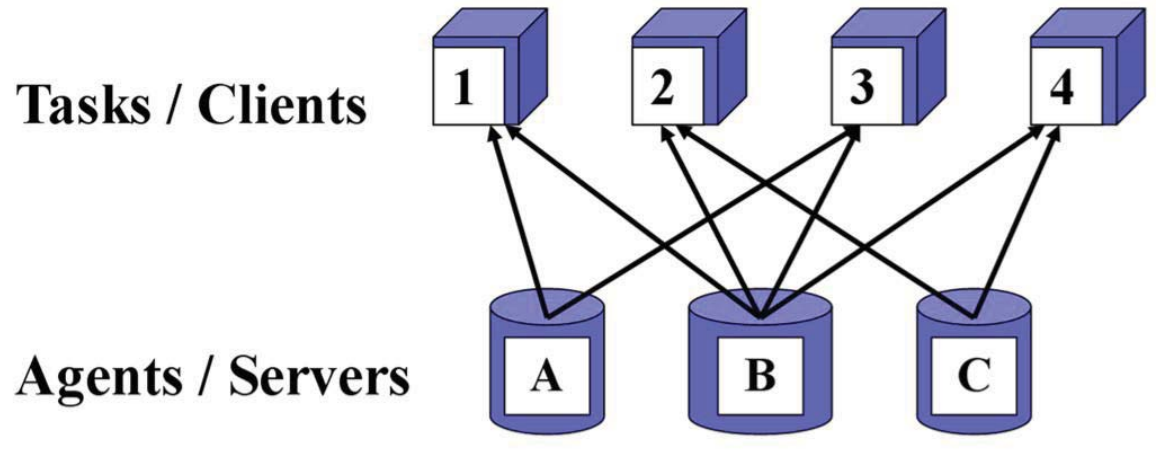

Figure 1. A simple client-server network topology displaying the pleiotropy and redundancy of the servers, which leads to a network topology of good robustness and low costs. Servers A, B, and C are shown to be servicing clients or performing tasks 1, 2, 3, and 4. An example of pleiotropy is server $\mathrm{B}$, which is shown to be serving all four clients concurrently. In contrast, an example of redundancy is task 4 that is shown to be performed by servers $\mathrm{B}$ and $\mathrm{C}$.

This is because evolutionary computation is heuristic, and any set of selected cost functions is only an approximation of the telecommunications network provider's preferences. It is difficult to accurately model all objectives in the evolutionary algorithm since subtle desired qualities of the network are difficult to formulate. Consequently, the solution obtained from optimizing a population of telecommunications nodes and links using evolutionary computation is not unique, and there is as much justification for the use of heuristic algorithms as exact optimization methods. ${ }^{10}$ The authors note the existence of other methods to optimize generic telecommunications networks. ${ }^{11-13}$ However, a detailed review of these methodologies is beyond the scope of this paper.

Hence, we intend to use evolutionary computation to optimize a generic telecommunications network using multiobjectives that a telecommunications service provider may consider employing. This enables us to model the network design process more accurately, where several conflicting goals of network optimization are not rare. For example, the telecommunications service provider may desire the network to be very reliable, provide multimedia content at high data rates, cover a large geographical area, and provide telecommunications services at affordable prices. It is desired that the outcomes of this ongoing research can facilitate the reduction of the installation, operating, and maintenance costs of the telecommunications service provider. Generic telecommunications networks within a layer in a network reference model is considered for optimization since it is difficult to obtain data for any specific network, as such information are considered proprietary to telecommunications service providers. An example of a network reference model is the International Organization for Standardization's (ISO) open systems interconnection (OSI) seven-layer communication architecture reference model. ${ }^{14,15}$

Finally, the connections and disconnections between servers and clients are part of a distributed, asynchronous, complex, and uninterrupted process that appears to be random to external observers. ${ }^{16}$ These connections and disconnections will influence the pleiotropy and redundancy of the telecommunications network and hence their trade-off. By appropriately modeling the dynamics of links addition and removal for different types of telecommunications networks, substantial advancements in scientific network research can be made. Subsequently, commercial firms in the telecommunications industry may profit from these by reducing their operating costs.

To our knowledge, there has hardly been any work done on studying the trade-off between pleiotropy and redundancy in telecommunications networks. Previous work included the use of the following fitness function $F^{5,6}$ :

$$
F=\frac{R_{e}}{C_{o}}
$$

where $F$ is the fitness function of the telecommunications network, $R_{e}$ is its reliability function, and $C_{o}$ is its cost function. This fitness function depends on the connectivity of the network to determine its reliability that sets the limit on the minimum cost. Therefore, when the cost is minimized and the reliability of the network is maximized, the fitness of the telecommunications network will not tend towards infinity. This implies that poorly connected networks are more costly 
than well connected networks. However, it does not take into account the self-organizing capabilities of the entities in the telecommunications network to reconfigure themselves so that they can still connect to the network.

The aim of this paper is to model symbiosis as a genetic operator for a steady state evolutionary algorithm that is used to optimize telecommunications networks within a layer in a network reference model. This allows the trade-off between pleiotropy and redundancy in telecommunications networks to be investigated as they evolve.

The paper is organized as follows. The next section discussed the proposed approach to optimize the telecommunications networks using an evolutionary algorithm. In Section 3, results from experiments are discussed. Section 4 outlines future work on improving our evolutionary algorithm. Finally, we draw conclusions from this work in Section 5.

\section{PROPOSED APPROACH}

In this section, a formulation for optimizing a generic telecommunications network in generalized goal programming terms and an evolutionary algorithm are provided. These will be used to determine the best possible solution based on the selected cost functions for this multiobjective optimization problem.

\subsection{Steady State Evolutionary Algorithm}

The steady state evolutionary algorithm is selected for the optimization process since it maintains the diversity of the population very well, and is good at retaining fitter members of the population. ${ }^{17}$ It does not cause rapid changes in population diversity or average fitness value of the population. This is because it generates a fixed number of offspring each generation, and only replaces a constant small number of chromosomes each generation. Consequently, this requires a large population to provide a diverse search space. ${ }^{9}$

A population of chromosomes is initialized at the start of the evolutionary algorithm, and a random number of edges is added at random to each chromosome. As the population evolves, edges are added to or removed from each selected chromosome after the mating process, during mutation, and the modeling of beneficial symbiotic relationships between telecommunications networks. At each generation, tournament selection is carried out only once to speed up the evolutionary process. ${ }^{18,19}$ A pseudo-random generated number will be compared with the probability of crossover to determine if the selected chromosomes should mate. If the former is smaller, the pair shall mate and produce one or two offspring.

Subsequently, another pseudo-random generated number will be compared with the probability of symbiosis. If the former is smaller, these fitter chromosomes, or their offspring if any were born, will interact and cooperate with each other. Finally, a pseudo-random generated number will be compared with the probability of mutation to determine if these chromosomes should mutate. Once again, they will mutate if the third pseudo-random generated number is smaller. The resultant mutants are then inserted back into the population via replacement of the chromosomes that have fitness values closest to theirs. ${ }^{9}$

The terms individuals, chromosomes, graphs, and telecommunications networks are used interchangeably. Similarly, the terms set and population of chromosomes are used synonymously. This is a consequence of modeling a telecommunications network as a directed graph $\mathrm{G}=\left(N_{\text {set }}, E_{\text {set }}\right)$, where $N_{\text {set }}$ is the set of nodes and $E_{\text {set }}$ is the set of directed edges. ${ }^{20}$ The nodes denote the geographical locations of the clients and servers in the network whilst the edges represent transmission, or data, links between clients and servers/other clients.

\subsection{Chromosome Representation}

In each chromosome, the topology of the network can be represented as an adjacency matrix if only one directed edge or none is allowed to be connected from node $A$ to node $B$. That is, the number of directed edges going from $A$ to $B$ is either one or zero, and the number of directed edges going from $B$ to $A$ can be one or zero. This is because parallel edges cannot be represented in adjacency matrices. ${ }^{21}$ By using adjacency matrices, storage space in memory can be saved by storing numerical values that represent the edge cost connecting any two nodes instead of node objects. The node objects belong to a software package that models the network topology as a graph. However, savings in memory storage comes at the expense of increased complexity and computation time. This bodes well since the focus is on meeting the constraints in the memory capacities of the computers that the simulations are run on, as opposed to the duration of simulations.

The adjacency matrix, which need not be symmetric, of a directed graph $G$ is the $n \cdot n$ matrix $A=\left[a_{i j}\right]$, where $a_{i j}$ represents the cost of the data link connecting node $i$ to node $j{ }^{21,22}$ Note that a symmetric matrix is used to represent a undirected graph. If there are no data links connecting node $i$ to node $j, a_{i j}$ is infinite. This implies that node $i$ cannot 
The Network

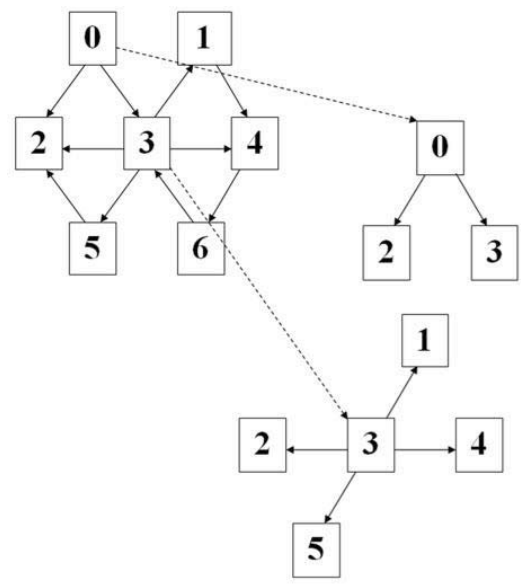

The Chromosome

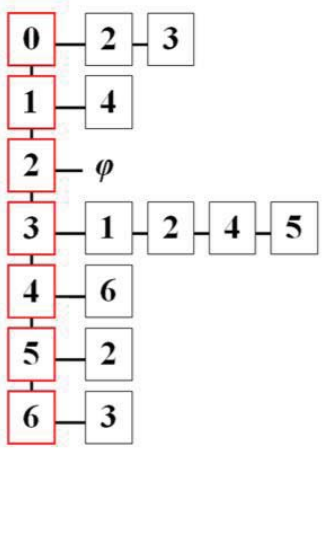

Figure 2. A representation of the telecommunications network topology as a list of adjacency lists for each node. The list of nodes in the network is given by the column highlighted on the left side of the chromosome. For each of these nodes, an adjacency list (horizontal row) is used to indicate destination nodes of edges starting from it. For example, node 3 has 4 outgoing edges; node 3 connects to nodes $1,2,4$, and 5 . Here, $\varphi$ indicates that node 2 has no outgoing edges.

connect to node $j$. Here, $a_{i i}$ represents any diagonal entry of $A$; it is zero. This is because it is not economically viable for the telecommunications service providers to install, operate, and maintain a data link that connects a node to itself since there is no information that can be gained by the node from this data link. For each node, an adjacency list is kept to store the destination nodes; see Fig. 2.

\subsection{Implementation of Genetic Operators}

A description for the basic models of biological phenomena employed in the optimization of telecommunications networks within a network layer is provided.

\subsubsection{Methods of selection.}

Tournament selection was used to select a pair of chromosomes from the population for the possibility of mating, symbiosis interaction, and mutation. A pair of chromosomes are picked at random using a pseudo-random number generator that follows a uniform distribution. The fitter chromosome of the pair is kept for genetic modification via mating, mutation, and the modeling of symbiosis. Another pair of chromosomes is selected at random, and the fitter chromosome is picked again to join the other fitter chromosome to form the mating pool. ${ }^{18,19}$ The selective pressure, which is measured by the difference between the average fitness of the mating pool and the average population fitness, can be adjusted with the number of chromosomes selected from the population (tournament size). This allows the selection operator to be more efficient and robust whilst overcoming the problems associated with scaling the fitness of each chromosome for every cost function. $8,9,23$

\subsubsection{Mating (Uniform Crossover).}

To get two chromosomes to mate, uniform crossover points are set at the boundaries between cells (nodes in the network) of the chromosome; all chromosomes are of the same length. The edges of every other node are swapped between the two chromosomes; see Fig. 3. Uniform crossover is used to decrease positional bias in the crossover operation. ${ }^{9,24,25}$

\subsubsection{Mutation.}

Chromosome selected for mutation undergo the following process: A pseudo-random number is generated to determine the number of nodes in a network that will be affected. For each affected node, another pseudo-random number will be generated to determine if an edge will be removed from or added to the network. If the second pseudo-random number is greater than a threshold, an edge will be added; else, an edge will be removed. See Fig. 4 for an example. 

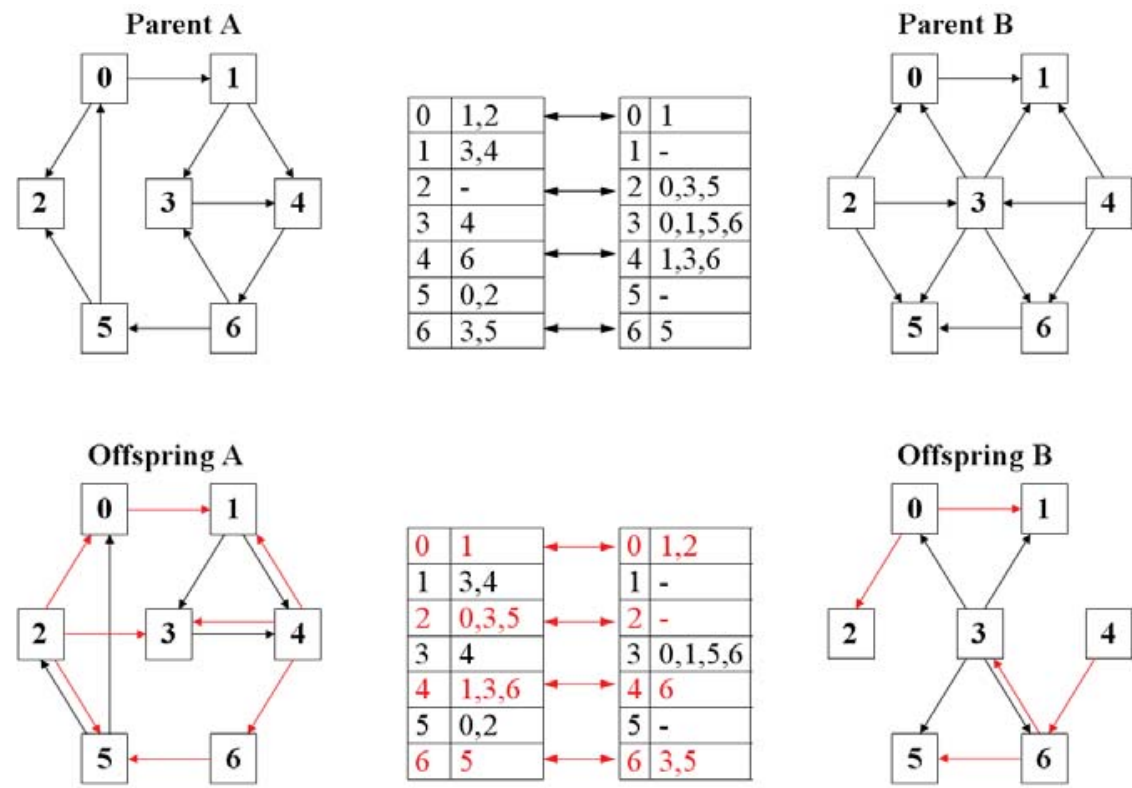

Figure 3. Mating process for pair of selected chromosomes; alternate nodes of the two chromosomes lying have their adjacency lists swapped. That is, crossover points are set between each pair of nodes. For example, node 4 of parent A is connected to node 6 , whilst node 4 of parent B is connected to nodes 1,3, and 6 . After performing uniform crossover on the pair that involves swapping the adjacency lists of every even indexed node, node 4 of offspring A is connected to nodes 1,3 , and 6 , whilst node 4 of offspring B is connected to node 1 .
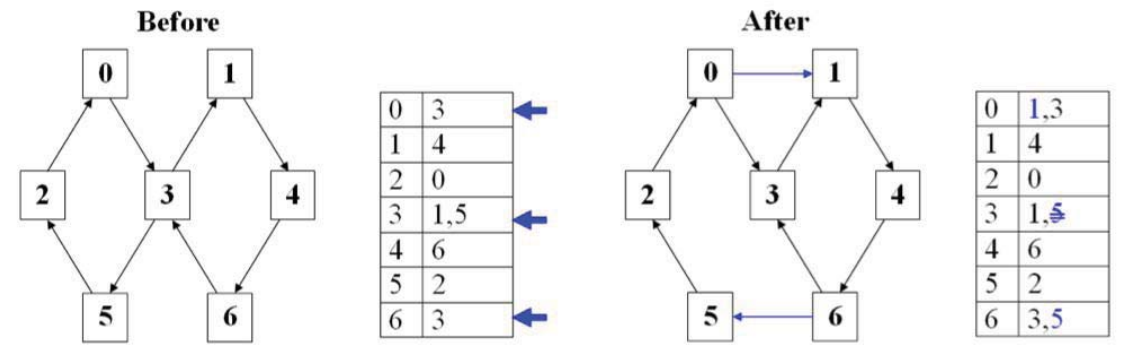

Figure 4. Random edges are added or removed by adding/removing destination nodes from a set of randomly selected adjacency lists. For example, node 3 has an outgoing edge to node 5 removed, whilst node 0 has added an outgoing edge to node 1, and node 6 has added an outgoing edge to node 5 .

\subsubsection{Symbiosis.}

In modeling beneficial symbiotic relationships, commensalism is considered as five nodes in a network with the largest amount of clustering coefficiency are selected, and a union operation of the edges belonging to this node and its corresponding node in another network is performed. Each of network in the population has the same set of nodes. This allows the network to improve its reliability by increasing the number of nodes with greater redundancy. The roles of these chromosomes are swapped as the process is repeated to model mutualism; see Fig. 5 for an example of mutualism.

Symbiosis allows the modeling of interaction between different telecommunications service providers (Telcos) within the same network layer. If a client is unable to obtain adequate reception from the client's Telco (Telco A) in a remote area, another Telco (Telco B) that collaborates with Telco A may provide routing of messages and signals between Telco A and the client. When Telco A provides similar services for Telco B's clients, each Telco requires less servers to cover the same geographical region. Thus, Telcos A and B will be able to set up their network at lower cost for the same reliability. 

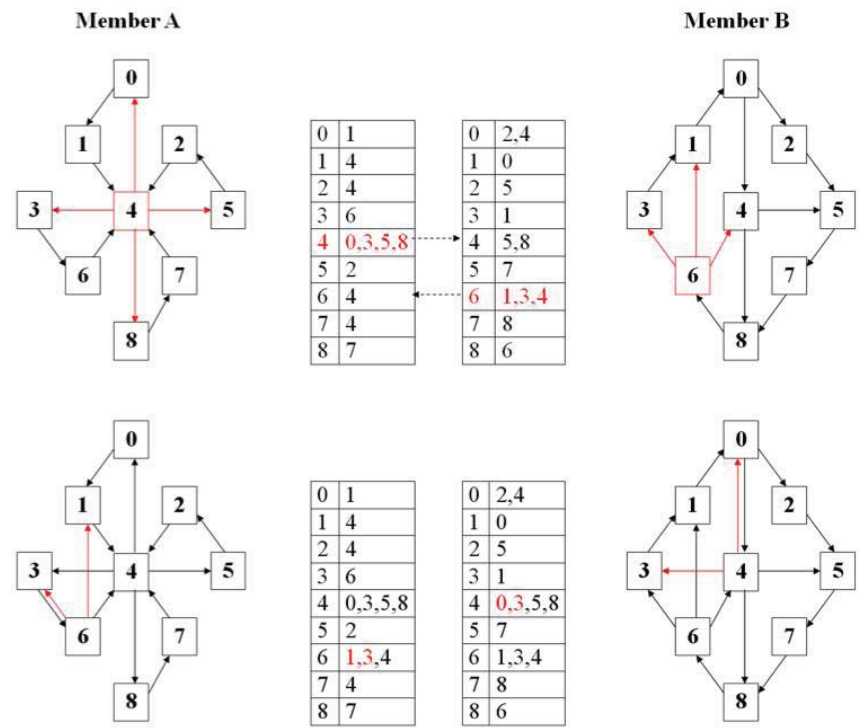

Figure 5. Modeling mutualism for pair of selected chromosomes. In this example, the node with the highest clustering coefficient is selected from member A and B; these nodes are node 4 and node 6 for member A and member B respectively. The adjacency lists of node 4 in member A and member B undergo a union operation, and the result is placed in member B. Similarly, union operation is performed on the adjacency lists of node 6 in member $\mathrm{A}$ and member $\mathrm{B}$; the result is placed in member $\mathrm{A}$.

\subsection{Metrics for measuring Pleiotropy and Redundancy}

Metrics for measuring pleiotropy and redundancy are provided as follows:

The metric used for measuring the pleiotropy of a server is:

$$
P_{\text {leio }}=\frac{\sum O_{\mathrm{s}}}{|S|}
$$

where $O_{\mathrm{s}}$ is the outgoing edge of a server, $\sum O_{\mathrm{s}}$ is the sum of $O_{\mathrm{s}}$ for each server, and $|S|$ is the number of servers in the telecommunications network.

The redundancy of a server can be measured as:

$$
R_{\text {edun }}=\frac{\sum I_{\mathrm{c}, \mathrm{s}}}{|C|},
$$

where $I_{c, s}$ is the incoming edge of a client with a server node as its source node, $\sum I_{c, s}$ is the sum of $I_{c, s}$ for each client, and $|C|$ number of clients in the telecommunications network

\subsection{Objective Functions}

Three objective functions, more specifically cost functions, are proposed to optimize the telecommunications network. The first cost function measures the total cost of all edges in the minimum spanning tree. ${ }^{26}$ Dijkstra's algorithm was used to determine the set of shortest paths for each node, where the sets were used to construct the set of shortest paths, to yield a cost function,

$$
C_{o}=\sum_{i \in \mathrm{mst}} E_{\mathrm{mst}_{i}}
$$

where $C_{o}$ is the cost function of the telecommunications network, mst refers to the set of edges belonging to the minimum spanning tree, and $E_{\mathrm{mst}_{i}}$ is the $i^{t h}$ edge in this set of edges.

A telecommunications network installed using a topology that is a set of shortest paths enables all the clients and servers to be connected using the least cost of all data links, which is determined by adding up the cost of each data link 
in that telecommunications network. Note that costs here refer to the costs of purchasing and installing the data links, and the propagation delay of the data links. The delay of a data link is the duration taken for a packet of data to travel along that link. ${ }^{14}$ Since data links are installed in telecommunications network as a wireless communications channel of a specified bandwidth, transmission line, or optical cable, this results in minimizing the installation costs of the network whilst allowing users of the telecommunications network to enjoy a good performance of data transmission. ${ }^{15}$

Given that the shortest path for any node in a graph can be determined from the set of shortest paths, the set of shortest paths can be determined as follows: Dijkstra's algorithm is applied to each node in the graph. An adjacency matrix $A$ is used to keep the shortest path for each node. The initial adjacency matrix $A_{i}$ of the graph $G$ prior to applying Dijkstra's algorithm is compared with the adjacency matrix $A_{d}$ obtained from applying Dijkstra's algorithm to every node. ${ }^{5,6}$ If $a_{i j}$ in $A_{i}$ is same as that in $A_{d}, a_{i j}$ is entered into the adjacency matrix $A_{m}$ of the set of shortest paths. This is because the set of shortest paths gives the shortest path from node $i$ to node $j$.

The next cost function measures the average degree of separation between two entities in the telecommunications network, ${ }^{27,28}$

$$
C_{o}=\frac{1}{\left|N_{\mathrm{set}}\right|} \cdot \sum_{i \in\left|N_{\mathrm{set}}\right|} D_{o S},
$$

where $\left|N_{\text {set }}\right|$ is the number of nodes in the graph $G$ and $D_{o S}$ is the degree of separation between any two nodes, which is the number of links connecting them. These number of links can be determined from assigning each link (where the costs is neither zero or infinity) in $A_{\text {adj }}$ to unit cost. By minimizing the average degree of separation, the link propagation delay will be reduced.

The last cost function measures the average clustering coefficient of servers in the telecommunications network, ${ }^{27,29}$

$$
C_{o}=\frac{1}{N_{2}} \cdot \sum_{i} \frac{2 \cdot E_{i}}{k_{i} \cdot\left(k_{i}-1\right)},
$$

where $N_{2}$ refers to the number of network nodes with more than or equal to two neighbors, $E_{i}$ is the number of connections, and $k_{i}$ is the number of neighbors of the node. The neighbors of a node are the nodes connected to it. The greater the average clustering coefficient of servers, the greater will be their ease to connect to more clients in the network as their neighbors would be more interconnected with each other. However, nodes with large clustering coefficiencies, which are also network hubs, also pose a threat to the reliability of the network in cascading failures of servers in the telecommunications network. ${ }^{4}$

The overall objective fitness function is used to determine how good the solution is for this problem described by the models employed. ${ }^{23}$ A scalar overall objective function obtained with a linear combination of weighted objectives requires knowledge about each of the aforementioned cost functions to determine its weight. This also results in a loss of information due to the absence of knowledge about their correlation and interaction. Hence, the Pythagorean sum is used; this requires the individual cost functions to be independent or lowly correlated to each other. The multi-cost function model is:

$$
C_{\text {all }}=\sqrt{\sum C_{o_{i}}^{2}}
$$

where $C_{\text {all }}$ is the overall fitness of the chromosome, and $C_{o_{i}}$ is the $i^{\text {th }}$ cost function.

\section{EXPERIMENTS AND RESULTS}

Simulation runs were carried out for each objective function, and their cross-correlation was determined. That is, the population of networks were optimized for one objective function during each simulation run. The values for the correlation were: The correlation for cost functions 1 and 2 is -0.1260 , and that for cost functions 1 and 3 is 0.2882 . For cost functions 2 and 3, the correlation is: 0.1772 . The P-values for these correlations are zero because of the large number of samples (1900) used and the floating point unit's inability to deal with very low numbers. Hence, we conclude that the correlations are statistically significant and the cost functions are independent of each other.

In our experiments, the values for the probabilities of crossover, symbiosis, and mutation are $0.26,0.23$, and 0.12 respectively. Values for the first two probabilities were higher than the probability of mutation so that the convergence time is not too quick and there is a reasonable amount of population diversity. Each of the 80 telecommunications networks 


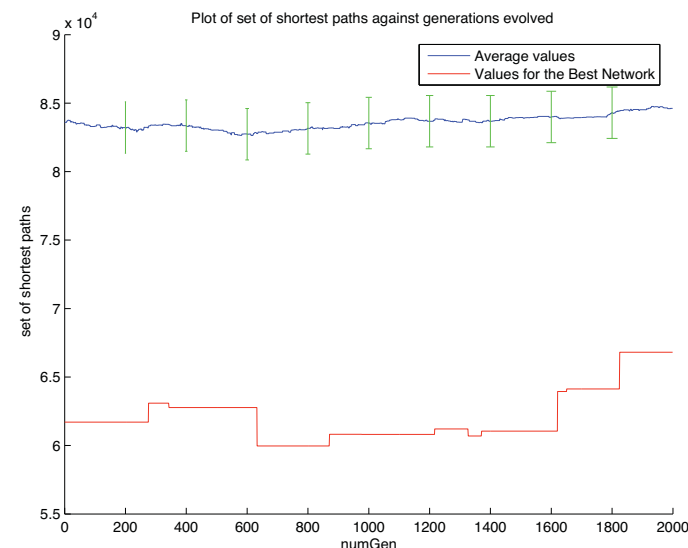

Figure 6. Plot of cost of minimum spanning tree against generations evolved, without symbiosis. Simulating the evolutionary optimization process without symbiosis for the same number of generations as when symbiosis is modeled, the cost of the minimum spanning tree for the fittest chromosome has yet to converge on an optimum level for a significant period of time. It is also observed that the best value for cost is in the order of 10 standard deviations below the average.

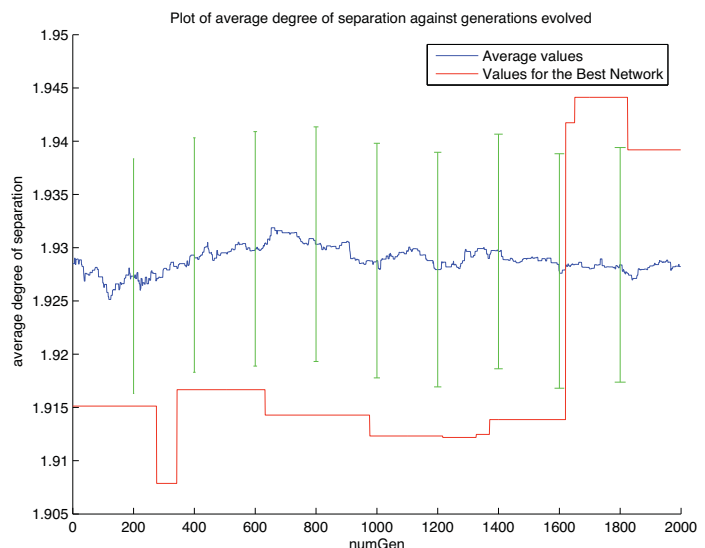

Figure 7. Plot of average degree of separation against generations evolved, without symbiosis. The average degree of separation for the fittest chromosome has risen sharply to its maximum value towards the end of the simulation; it takes a long time to converge around an optimum. Its average values, just like those of the cost of minimum spanning tree, do not vary much due to the application of the steady-state algorithm. The cost of the best network is observed to exceed the average cost of the population due to the interaction between the cost functions.

in the population had 75 clients and 10 servers; the memory capacity of our computing resources limits the number of nodes that can be simulated in a network. These experiments were run for 2000 generations with and without symbiosis; the probability of symbiosis is set to 0.0 when symbiosis is not modeled. The simulation results are indicated as shown in Figures 6-13.

The average value for the overall fitness of the population increased marginally near the end of the simulation without symbiosis due to the implementation of the steady state evolutionary algorithm. Since only two chromosomes are selected at each generation, hardly any significant changes were made to the population of chromosomes. The pleiotropy and redundancy values of servers and clients in the fittest telecommunications network were constant when the symbiosis genetic operator was not applied. The convergence times for each cost functions evaluated with were also slow; the values for each cost function did not change much after 1700 generations, see Figures 6-8. It is also observed that the best value for cost is in the order of 10 standard deviations below the average.

The first two cost functions, see equations (4) and (5), were found to be highly and negatively correlated with the third cost function, see equation (6); this may be an example of Parrondo's paradox occurring. ${ }^{30,31}$ The correlation for cost functions 1 and 2 is 0.9807 , and the correlation for cost functions 1 and 3 is -0.9987 and that for cost functions 2 and 3 is -0.9874. Once again, the values for correlation have P-values of zero for the aforementioned reasons. When symbiosis is not modeled, the average clustering coefficiencies of the optimum network in the population decreases as the networks are optimized due to the falling number of edges in the optimum network. This is because as the average degree of separation between any two nodes in the network is minimized with the number of edges needed to form a minimum spanning tree, edges are randomly removed from the network. This also implies that with a smaller number of edges, more edges may be needed to connect any two nodes. This explains why the values for the first two cost functions have increased. Thus, whilst the optimum network is less susceptible to cascading failures of servers, it is more costly as the optimum network would have a larger minimum spanning tree and average degree of separation between any two nodes. The last cost function also has a greater impact than the first two since it is minimized as desired, whilst the other two cost functions are maximized due to their high negative correlation.

The pleiotropy values for servers were found to have increased and optimized when the symbiosis genetic operator was applied, whilst the values of redundancy for clients remained constant; see Fig. 9. The application of the symbiosis genetic operator also reduced the convergence time significantly for each cost function; the values for each cost function and the overall cost of the fittest chromosome did not change much after 500 generations, see Figures 9-13. 


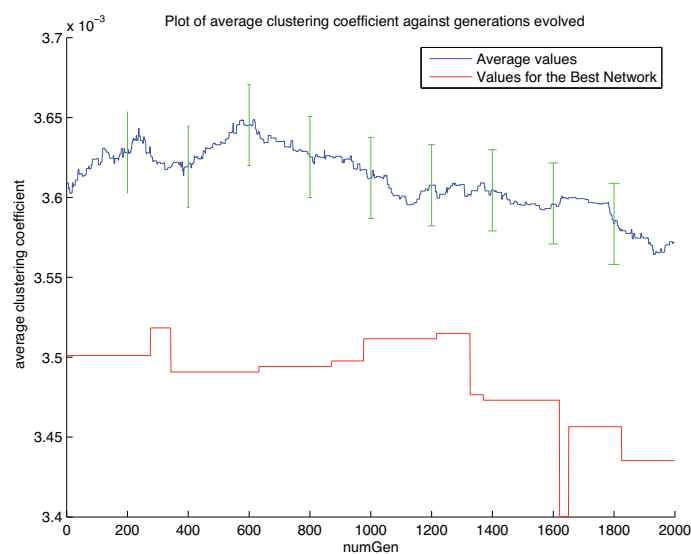

Figure 8. Plot of average clustering coefficient against generations evolved, without symbiosis. The average clustering coefficient for the fittest chromosome is decreasing. This indicates a high negative correlation between this cost function and the other two. This may be an example of Parrondo's paradox. As the networks are optimized, the number of edges in the graph will decrease as edges are randomly removed. Hence, the average clustering coefficient of the optimum network will decrease, as the values for the first two cost functions increase due to the difficulty in connecting any two nodes. Since its cost values are decreasing as desired, it has a bigger impact in the overall cost of the network than the other two cost functions. As before, the cost of the best network is observed to exceed the average cost of the population due to the interaction between the cost functions.

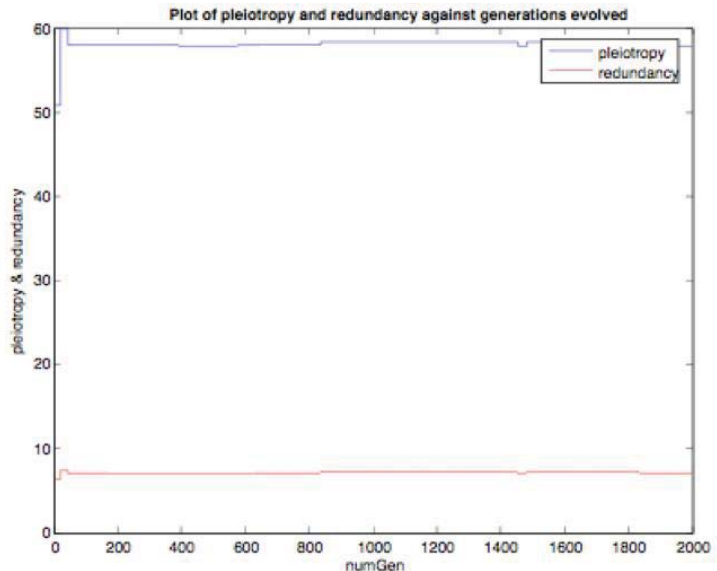

Figure 9. Plot of pleiotropy and redundancy against generations evolved, with symbiosis. The pleiotropy of servers has increased, and converges on an optimum rapidly, when symbiosis is modeled in the evolutionary algorithm.

The first cost function has a significant negative correlation with the last two cost functions; the values for correlation are -0.4110 and -0.9945 with P-values of $2.4968 \times 10^{-78}$ and 0.0 . The correlation between the cost functions 2 and 3 is 0.3240 and the $\mathrm{P}$-value is $1.0668 \times 10^{-47}$. As the minimum spanning tree and the average degree of separation is minimized, the number of edges in the optimum network are increased due to the modeling of beneficial symbiotic relationships between networks. This is because whenever a symbiotic operation is performed, a union of the adjacency lists of a few nodes with very high clustering coefficiency is carried out. Thus, more nodes can be connected to each server, and this increases the average clustering coefficiency. In addition, the first two cost functions have a greater impact than this cost function, which is maximized instead of minimized. Consequently, the optimum network would have a smaller minimum spanning tree and average degree of separation between any two nodes. Finally, the authors believe that the cost of the best network is observed to exceed the average cost of the population, as shown in Figures 7 and 12, due to the interaction between the cost functions.

\section{FUTURE WORK}

Avenues that are worth exploring include the following: Improvement can be made to simultaneously satisfy competing design objectives by considering multi-objective optimization with evolutionary algorithms. ${ }^{32}$ For example, Pareto-based multiobjective evolutionary algorithms can be considered since it does not combine the objectives to derive the fitness values of the solutions; it obtains the fitness values of solutions by comparing the respective objective vectors projected into their objective space. ${ }^{23}$

Parasitic relationships can also be modeled in the evolution of the telecommunications networks. The authors believe this would allow the population to be more diverse and take a longer time for the population to converge at an optimal solution. However, the authors note that it is natural for entities (individuals, firms, and governmental organizations) in social-economic networks and organisms in biological networks to avoid parasites. Hence, a chromosome should keep a list of previous intolerable partners that it would prefer not enter a relationship with again. This allows former parasitic 


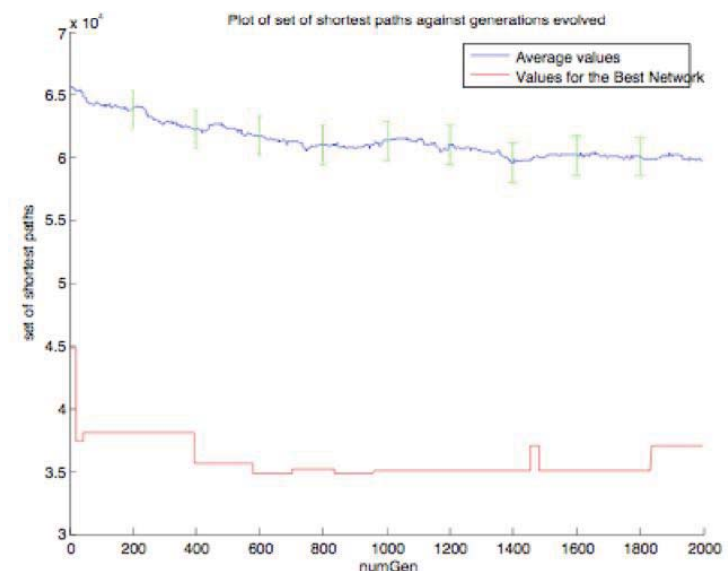

Figure 10. Plot of cost of minimum spanning tree against generations evolved, with symbiosis. The cost of minimum spanning tree for the fittest chromosome has converged significantly faster with symbiosis, compare this with Fig. 6 .

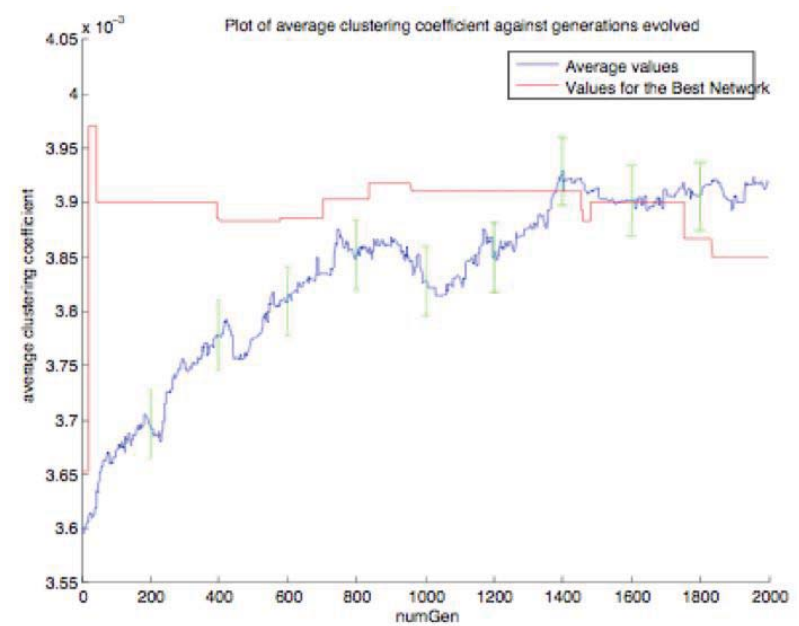

Figure 12. Plot of average clustering coefficient against generations evolved, with symbiosis. Once again, a high negative correlation between this cost function and the other two is observed. This may also be an example of Parrondo's paradox. This is because as the minimum spanning tree and the average degree of separation is minimized, the number of edges in the optimum network are increased due to the modeling of beneficial symbiotic relationships between networks. Thus, more nodes can be connected to each server.

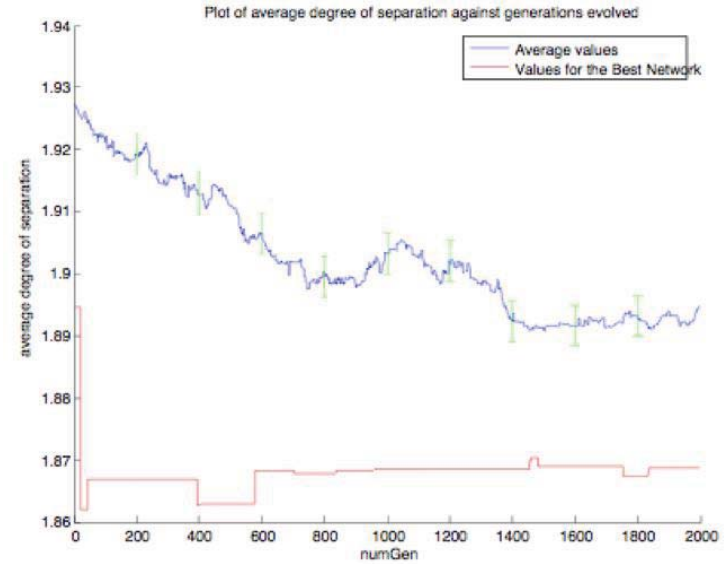

Figure 11. Plot of average degree of separation against generations evolved, with symbiosis. The average degree of separation for the fittest chromosome has also converged significantly faster with symbiosis, compare this with Fig. 7.

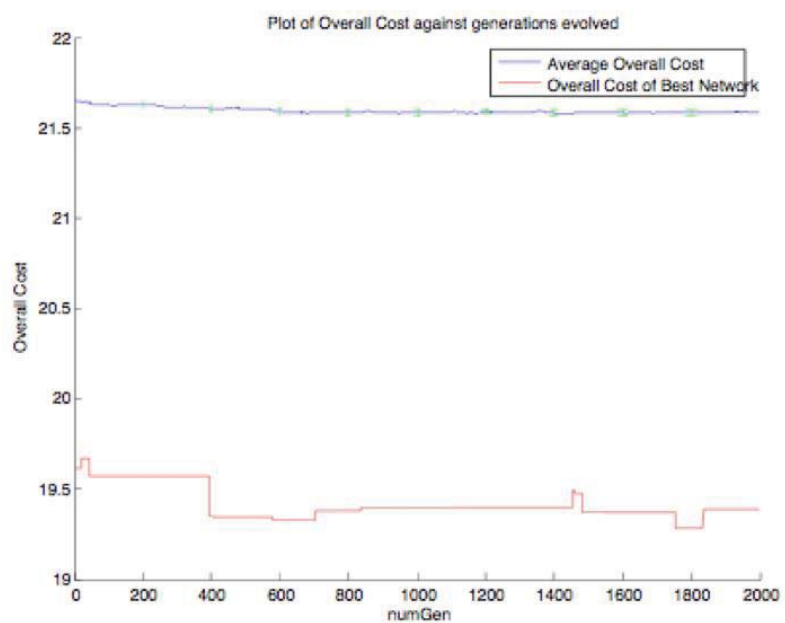

Figure 13. Plot of overall cost against generations evolved, with symbiosis. The overall cost of the fittest chromosome when symbiosis is modeled is marginally minimized.

chromosomes to renew relationships with previous partners as current commensal or mutualistic partners. Consequently, the objective of parasitic chromosomes is to convince its hosts that they are not currently parasitic. ${ }^{33}$

\section{CONCLUSIONS}

Telecommunications networks were optimized using steady state evolutionary algorithms, with and without symbiosis modeled as a genetic operator. The application of symbiosis as a genetic operator increases the pleiotropy of servers in the 
telecommunications network; thus, making the networks less expensive for the same amount of robustness. In addition, modeling symbiosis also significantly reduce convergence time during optimization with evolutionary computation. The authors expect this analysis to be most useful for the physical, data link, and network layers of the International Organization for Standardizations (ISO) open systems interconnection (OSI) communication architecture reference model.

\section{ACKNOWLEDGMENTS}

The authors would like to thank Zbigniew Michalewicz and Andrew Allison, from the University of Adelaide, for useful discussions on evolutionary computation and network models.

\section{REFERENCES}

1. R. Albert and A.-L. Barabási, "Topology of evolving networks: Local events and universality," Physical Review Letters 85, pp. 5234-5237, December 2000.

2. D. Wilkinson, "Civilizations as networks: Trade, war, diplomacy, and command-control: States-systems bonded by influence, alliance, and war relations," Complexity 8, pp. 82-86, September - October 2002.

3. M. Amin, "Toward self-healing infrastructure systems," Computer 33, pp. 44-53, August 2000.

4. D. Newth and J. Ash, "Evolving cascading failure resilience in complex networks," in Proceedings of the Eighth Asia-Pacific Symposium on Intelligent and Evolutionary Systems, G. Goos, J. Hartmanis, and J. van Leeuwen, eds., 2723, pp. 125-136, (Cairns, Australia), 6-7 December 2003.

5. M. J. Berryman, W.-L. Khoo, H. Nguyen, E. O’Neill, A. Allison, and D. Abbott, "Exploring tradeoffs in pleiotropy and redundancy using evolutionary computating," in Proceedings of SPIE: BioMEMS and Nanotechnology, D. V. Nicolau, ed., 5275, pp. 49-58, (Perth, Australia), 10-12 December 2003.

6. M. J. Berryman, A. Allison, and D. Abbott, "Optimizing genetic algorithm strategies for evolving networks," in Proceedings of SPIE: Noise in Communication, L. B. White, ed., 5473, pp. 122-130, (Maspalomas, Gran Canaria, Spain), 26-27 May 2004.

7. S. Obayashi, "Pareto genetic algorithm for aerodynamic design using the navier-stokes equations," in Genetic Algorithms and Evolution Strategy in Engineering and Computer Science: Recent Advances and Industrial Applications, D. Quagliarella, J. Périaux, C. Poloni, and G. Winter, eds., ch. 12, pp. 245-266, John Wiley \& Sons, Chichester, West Sussex, England, 1998.

8. C. Poloni and V. Pediroda, "GA coupled with computationally expensive simulations: tools to improver efficiency," in Genetic Algorithms and Evolution Strategy in Engineering and Computer Science: Recent Advances and Industrial Applications, D. Quagliarella, J. Périaux, C. Poloni, and G. Winter, eds., ch. 13, pp. 267-288, John Wiley \& Sons, Chichester, West Sussex, England, 1998.

9. G. Winter, M. Galán, P. Cuesta, and D. Greiner, "Genetic algorithms: A stochastic improvement technique. tools, skills, pitfalls, and examples," in Genetic Algorithms in Engineering and Computer Science, G. Winter, J. Périaux, M. Galán, and P. Cuesta, eds., ch. 12, pp. 217-249, John Wiley \& Sons, Chichester, West Sussex, England, 1995.

10. T. J. Stewart, R. Janssen, and M. van Herwijnen, "A genetic algorithm approach to multiobjective land use planning," Computers \& Operations Research 31, pp. 2293-2313, December 2004.

11. G. Di Caro and M. Dorigo, "Two ant colony algorithms for best-effort routing in datagram networks," in Proceedings of the Tenth IASTED International Conference on Parallel and Distributed Computing and Systems (PDCS'98), Y. Pan, S. G. Akl, and K. Li, eds., pp. 541-546, (Las Vegas, NV), 28-31 October 1998.

12. L. Li, Design and optimization of packet switching and traffic grooming in WDM optical networks. Ph.D. Thesis, University of Nebraska, Lincoln, Nebraska, December 2003.

13. M. S. Medrano, M. B. Trindade, N. S. A. de Chaves, M. D. Fernandez, and H. J. M. Filho, "An optimization model for MPLS networks," in NETWORKS 2004: 11th International Telecommunications Network Strategy and Planning Symposium, H. Kaindl, ed., pp. 285-290, (Vienna, Austria), 13-16 June 2004.

14. D. E. Comer, Computer Networks and Internets with Internet Applications, Pearson Prentice Hall, New Jersey, fourth ed., 2004.

15. W. Tomasi, Electronic Communications Systems: Fundamentals through Advanced, Prentice-Hall, New Jersey, fourth ed., 2001.

16. D. M. Pennock, G. W. Flake, S. Lawrence, E. J. Glover, and C. L. Giles, "Winners don't take all: Characterizing the competition for links on the web," Proceedings of the National Academy of Sciences 99, pp. 5207-5211, April 2002. 
17. D. Chafekar, J. Xuan, and K. Rasheed, "Constrained multi-objective optimization using steady state genetic algorithms," in Lecture Notes in Computer Science: Proceedings of the Genetic and Evolutionary Computation Conference GECCO 2003, G. Goos, J. Hartmanis, and J. van Leeuwen, eds., 2723, pp. 813-824, Springer-Verlag, (Berlin, Germany), July 2003.

18. B. L. Miller and D. E. Goldberg, "Genetic algorithms, tournament selection, and the varying effects of noise," Complex Systems 9(3), pp. 193-212, 1996.

19. E. Cantú-Paz, "Order statistics and selection methods of evolutionary algorithms," Information Processing Letters $\mathbf{8 2}$, pp. 15-22, April 2002.

20. P. Mirchandani and D. Simchi-Levi, "Networks and flows: Communication network design models," in Handbook of Graph Theory, J. L. Gross and J. Yellen, eds., Discrete Mathematics and its Applications, pp. 1117-1118, CRC Press, Boca Raton, FL, 2004.

21. N. Deo, Graph Theory with Applications to Engineering and Computer Science, Prentice-Hall, Inc., Englewood Cliffs, NJ, 1974.

22. V. K. Balakrishnan, Graph Theory, Schaum's Outline, McGraw-Hill, New York, NY, 1997.

23. J. Périaux, M. Sefrioui, and B. Mantel, "GA multiple objective optimization strategies for electromagnetic backscattering," in Genetic Algorithms and Evolution Strategy in Engineering and Computer Science: Recent Advances and Industrial Applications, D. Quagliarella, J. Périaux, C. Poloni, and G. Winter, eds., ch. 11, pp. 225-243, John Wiley \& Sons, Chichester, West Sussex, England, 1998.

24. L. J. Eshelman, R. A. Caruana, and J. D. Schaffer, "Biases in the crossover landscape," in Proceedings of the Third International Conference on Genetic Algorithms, J. D. Schaffer, ed., pp. 10-19, Morgan Kaufmann Publishers, Inc., (San Mateo, CA), 4-7 June 1989.

25. G. Syswerda, "Uniform crossover in genetic algorithms," in Proceedings of the Third International Conference on Genetic Algorithms, J. D. Schaffer, ed., pp. 2-9, Morgan Kaufmann Publishers, Inc., (San Mateo, CA), 4-7 June 1989.

26. M. T. Goodrich and R. Tamassia, Data Structures and Algorithms in Java, John Wiley \& Sons, Inc., New York, NY, second ed., 2001.

27. D. J. Watts, Six Degrees: The Science of a Connected Age, W. W. Norton \& Company, New York, NY, 2003.

28. A.-L. Barabási, Linked: How Everything Is Connected to Everything Else and What It Means for Business, Science, and Everyday Life, Plume, New York, NY, 2003.

29. P. O. Boykin and V. P. Roychowdhury, "Leveraging social networks to fight spam," IEEE Computer 38, pp. 61-68, April 2005.

30. G. P. Harmer and D. Abbott, "Losing strategies can win by parrondo's paradox," Nature 402, p. 864, Dec 1999.

31. G. P. Harmer and D. Abbott, "A review of parrondo's paradox," Fluctuation and Noise Letters 2, pp. R71-R107, June 2002.

32. A. Chipperfield and P. Fleming, "An overview of evolutionary algorithms for control systems engineering, www.shef.ac.uk/content/1/c6/03/34/64/ AnOverviewofEvolutionaryAlgorithmsforControlSystemsEngineering.pdf," 2003. Viewed 8 May, 2005.

33. P. H. Raven and G. B. Johnson, Biology, McGraw-Hill, New York, NY, sixth ed., 2002.

34. H. Kargupta, "Gene expression: The missing link in evolutionary computation," in Genetic Algorithms and Evolution Strategy in Engineering and Computer Science: Recent Advances and Industrial Applications, D. Quagliarella, J. Périaux, C. Poloni, and G. Winter, eds., ch. 4, pp. 59-83, John Wiley \& Sons, Chichester, West Sussex, England, 1998. 